\title{
Association of p73 G4C14-to-A4T14 polymorphism with non-small cell lung cancer risk
}

\author{
SHUANG SHUANG WANG ${ }^{1}$, XIANG QIN ZHU², SHAO DI YANG ${ }^{3}$, \\ LIN LI DONG ${ }^{1}$, WEN LI ${ }^{1}$ and JIANXIN TANG ${ }^{1}$
}

\begin{abstract}
${ }^{1}$ Key Laboratory of Green Packaging and Application of Biological Nanotechnology, Hunan University of Technology, Zhuzhou, Hunan 412008; ${ }^{2}$ Department of Pathology, Hunan Provincial Tumor Hospital, Changsha, Hunan 410113;

${ }^{3}$ Institute of Biomedical Engineering, School of Geosciences and Info-Physics,

Central South University, Changsha, Hunan 410006, P.R. China
\end{abstract}

Received August 16, 2014; Accepted April 29, 2015

DOI: $10.3892 / \mathrm{ol} .2015 .3322$

\begin{abstract}
The p73 gene is a structural and functional homolog of the p53 gene, which has a crucial role in mediating cell cycle arrest and apoptosis. Numerous previous studies have investigated the polymorphism of p73 G4C14-to-A4T14 at exon 2, as it was suggested to affect gene expression and result in functional significance. However, the correlation of this polymorphism with clinicopathological variables of patients with non-small cell lung cancer (NSCLC) remains to be elucidated. The aim of the present study was to examine the association between the gene polymorphism of p73 G4C14-to-A4T14 and the risk of developing NSCLC. The single-nucleotide polymorphisms of p73 G4C14-to-A4T14 were genotyped using polymerase chain reaction with confronting two-pair primers and direct DNA sequencing in 186 NSCLC patients and 196 cancer-free controls. $\chi^{2}$-tests and logistic regression analysis were used to analyze the experimental data, including the determination of odds ratio (OR), 95\% confidence intervals (95\% CIs) and P-values. The results demonstrated that the AT/AT genotype was associated with a significantly decreased risk of NSCLC $(\mathrm{P}=0.010 ; \mathrm{OR}=0.370 ; 95 \% \mathrm{CI}=0.170-0.806)$ compared with the $\mathrm{GC}$ allele genotypes including $\mathrm{GC} / \mathrm{GC}$ and GC/AT. In addition, carriers of the AT allele exhibited a significantly reduced risk of NSCLC $(\mathrm{P}=0.038 ; \mathrm{OR}=0.713 ; 95 \% \mathrm{CI}=0.517-0.983)$ compared with non-carriers. In conclusion, these results indicated that the p73 G4C14-to-A4T14 polymorphism was a potential marker of NSCLC genetic susceptibility. However,
\end{abstract}

Correspondence to: Dr Wen Li or Professor Jianxin Tang, Key Laboratory of Green Packaging and Application of Biological Nanotechnology, Hunan University of Technology, 88 Taishan Road, Zhuzhou, Hunan 412008, P.R. China

E-mail: liwenjoha@yahoo.com

E-mail: jianxin_tang@yahoo.com

Key words: p73G4C14-to-A4T14, polymorphisms, non-small cell lung cancer further studies with a larger population are required in order to confirm these results.

\section{Introduction}

Increasing evidence has confirmed that lung cancer, predominantly non-small cell lung cancer (NSCLC), is the primary cause of cancer-associated mortality worldwide, with a significantly increased risk among males (1). It has been well established that lung cancer is a complex disease that is highly associated with environmental pollutants and smoking in the general population; in addition, genetic factors are known to have an important role in this disease. Therefore, the study of genetic variants relevant to lung cancer may contribute to fully elucidating the pathogenesis of this malignancy, including its formation and development, as well as to predicting an individual's risk of developing NSCLC.

The p73 gene was identified at chromosome lp36.33 in 1997 (2), a site that has been demonstrated to commonly undergo loss of heterozygosity ( $\mathrm{LOH}$ ) in various types of cancer (2-4). $\mathrm{LOH}$ at the p73 gene has been reported to occur in $62 \%$ of lung cancer patients (5). The p73 protein is a structural and functional homolog of p53, a classic tumor suppressor (2). Numerous studies have demonstrated that the p73 gene had a crucial role in the pathogenesis of various types of cancer, including lung cancer (6-9). Therefore, it was hypothesized that p73 may be a potential susceptibility gene for lung cancer; in addition, it was suggested that p73 gene polymorphisms may results in differences in susceptibility to lung cancer (10).

The p73 gene contains a polymorphism in the $5^{\prime}$ untranslated region (UTR), which is located upstream of the initiating AUG codon in exon 2 of p73 gene; in theory, this region may have the ability to form a stem-loop structure and modulate susceptibility to cancer $(2,11,12)$. This polymorphism involves two single-nucleotide polymorphisms (SNPs) at positions 4 (G-A) and 14 (C-T) (2). As these SNPs are in complete linage equilibrium, only three genotypes are possible: $\mathrm{GC} / \mathrm{GC}$, GC/AT and AT/AT. Previous studies have reported an association between the G4C14-to-A4T14 polymorphism and the risk of developing various types of cancer. Li et al (13) revealed 
a marked association in an American population between the AT allele or genotype and the risk of developing squamous cell carcinoma of the head and neck (13); in addition, a study by Ryan et al (14) indicated that AT/AT homozygotes were significantly uncommon in esophageal cancer patients from an Irish population. Furthermore, Hu et al (15) reported an association between dinucleotide polymorphism of the p73 gene and a reduced risk of lung cancer in a Chinese population and Li et al (16) determined that the variant GC/AT and AT/AT genotypes were associated with a significantly increased risk of lung cancer (16). Associations between p73 gene polymorphisms and the risk of lung cancer therefore illustrate that the p73 gene has a critical role in lung cancer carcinogenesis (15-18). However, there have been inconsistencies in the data regarding these associations. Therefore, the present study aimed to perform a case-control study in order to examine whether the p73 G4C14-to-A4T14 polymorphism was associated with NSCLC susceptibility and its effects on the clinical characteristics of patients.

\section{Materials and methods}

Patients. A total of 184 consecutive lung cancer patients and 196 cancer-free controls were enrolled in the present study from the Hunan Provincial Tumor Hospital (Changsha, China) between January 2013 and June 2014. Patients included in the present study had been newly diagnosed with NSCLC following histopathological confirmation. At recruitment, each patient was interviewed to collect personal information regarding demographic characteristics factors, including age, gender, smoking status and histological type (Table I). Written informed consent was obtained from all participants and the study was performed with the approval of the Changsha Clinical Research Center Ethics Committee (Changsha, China).

Polymerase chain reaction (PCR) analysis. Blood samples $(400 \mu \mathrm{l})$ were collected from each participant and genomic DNA was extracted using a DNA extraction kit (Takara Biotechnology Co., Ltd., Dalian, China). Blood samples and DNA samples were stored at $-70^{\circ} \mathrm{C}$. DNA samples were then analyzed for the p73 G4C14-to-A4T14 polymorphism using PCR with confronting two-pair primers (PCR-CTPP; Sangon Biotech Co., Ltd., Shanghai, China). The PCR primers used were as follows (19): Forward (F)1, 5'-CCACGGATGGGT CTGATCC-3' and reverse (R)1, 5'-GGCCTCCAAGGGCAG CTT-3'; and F2, 5'-CCTTCCTTCCTGCAGAGCG-3' and R2, 5'-TTAGCCCAGCGAAGGTGG-3'. PCR was performed with a $15 \mu 1$ reaction mixture, containing $7.5 \mu 1$ Taq PCR Master Mix (Sangon Biotech Co., Ltd.), $2.5 \mu 1$ double-distilled $\mathrm{H}_{2} 0,1 \mu \mathrm{l}$ each of four primers and $1 \mu \mathrm{l}$ DNA template. PCR was performed using a Gene Amp PCR System 9700 (Applied Biosystems, Waltham, MA, USA) as follows: Initial denaturation at $95^{\circ} \mathrm{C}$ for $5 \mathrm{~min} ; 35$ cycles of $95^{\circ} \mathrm{C}$ for $40 \mathrm{sec}, 60^{\circ} \mathrm{C}$ for $40 \mathrm{sec}$ and $72^{\circ} \mathrm{C}$ for $40 \mathrm{sec}$; and a final extension at $72^{\circ} \mathrm{C}$ for 5 min (19). The PCR products were separated on a $2 \%$ agarose gel (Sangon Biotech Co., Ltd.) with ethidium bromide staining (Sinopharm Chemical Reagent Co., Ltd., Shanghai, China) and visualized using the Vilber Fusion FX7 gel-imaging system (11200238; Vilber Lourmat Deutchland, Eberharedzell, Germany).
Table I. Distributions of characteristics among non-small cell lung cancer cases and controls.

\begin{tabular}{|c|c|c|c|}
\hline & Cases $(\%)$ & Controls $(\%)$ & \\
\hline Variable & $\mathrm{n}=186$ & $\mathrm{n}=198$ & P-value \\
\hline Age & & & 0.678 \\
\hline$\leq 50$ & $90(48.3)$ & $100(50.5)$ & \\
\hline$>50$ & $96(51.6)$ & $98(49.5)$ & \\
\hline Gender & & & 0.837 \\
\hline Male & $137(73.7)$ & $144(72.9)$ & \\
\hline Female & $49(26.3)$ & $54(27.3)$ & \\
\hline Smoking status & & & $<0.001$ \\
\hline No & $67(36.0)$ & $125(63.1)$ & \\
\hline Yes & $119(64.0)$ & $73(36.9)$ & \\
\hline \multicolumn{4}{|l|}{ Histological type } \\
\hline Squamous carcinomas & $116(62.4)$ & & \\
\hline Adenocarcinomas & $58(31.2)$ & & \\
\hline Others & $12(6.4)$ & & \\
\hline
\end{tabular}

P-values were determined using a two-sided $\chi^{2}$-test.

Statistical analysis. Gene counting was used to determine the genotype and allele frequencies for p73 G4C14-to-A4T14 polymorphism using the $\chi^{2}$-test. Comparisons of age, sex and smoking status distributions between NSCLC cases and controls were performed using the $\chi^{2}$-test. The association between the p73 genotypes and NSCLC risk was assessed using the logistic regression model, while odds ratios (ORs), 95\% confidence intervals (95\% CIs) and P-values were also calculated. The association between $\mathrm{p} 73$ polymorphic variants and clinicopathological characteristics was determined using the $\chi^{2}$-test. $P<0.05$ was considered to indicate a statistically significant difference between values. All statistical analyses were performed using SPSS 17.0 software (SPSS Inc., Chicago, IL, USA).

\section{Results}

Characteristics of study subjects. In total, 186 NSCLC cases and 198 control subjects were recruited into the present study. The frequency distribution of the basic characteristics of the patients and controls is summarized in Table I. The distributions of age and sex seemed to be matched fully and there was no significant difference between cases and controls. However, there were a significantly increased number of smokers in the NSCLC group compared with the control group (64.0 vs. $36.9 \%$, respectively; $\mathrm{P}<0.001)$. This therefore indicated that smoking was a high risk factor associated with NSCLC in patients enrolled in the present study.

Distribution of $p 73$ polymorphisms. The association between p73 polymorphisms and susceptibility to NSCLC were explored using PCR-CTPP; as the two SNPs were in complete linage equilibrium, only three genotypes were possible: GC/GC, GC/AT and AT/AT. The electrophoresis results are shown in Fig. 1 and the sequencing results of PCR 


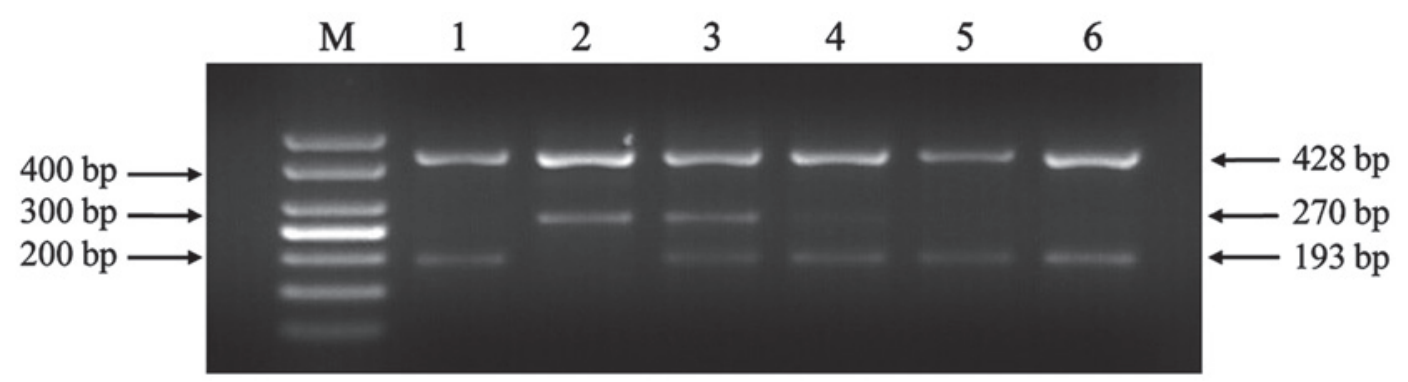

Figure 1. Detection of the p73 G4C14-to-A4T14 polymorphism by polymerase chain reaction-confronting two-pair primers. Lane M, 50 bp DNA ladder; lanes 1, 4, 5 and 6, GC/GC genotype; lane 2, AT/AT genotype; lane 3, GC/AT genotype.

A

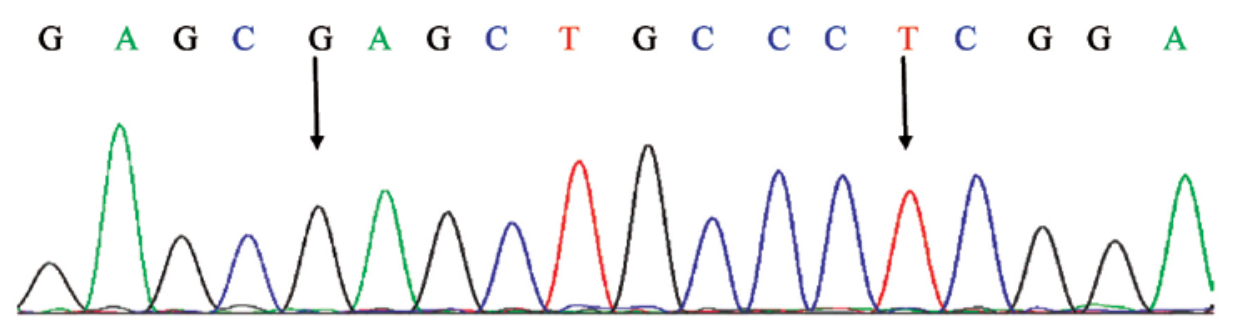

B

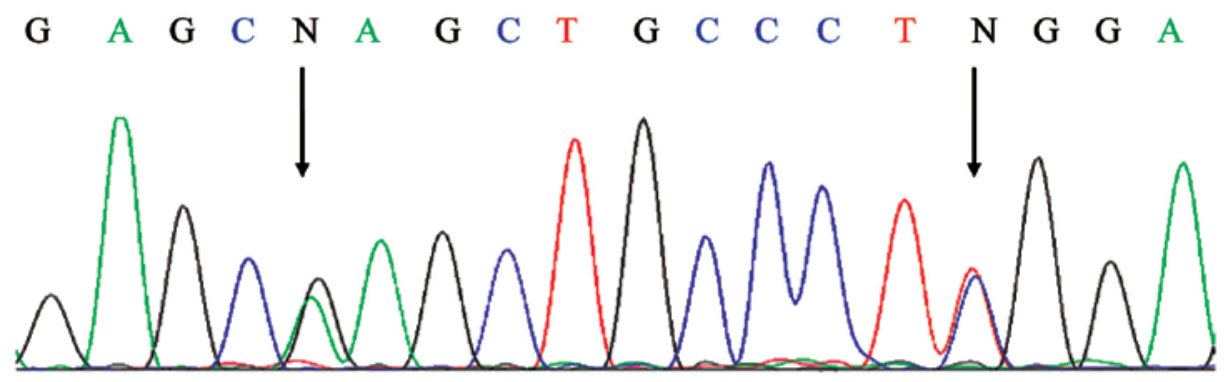

C



Figure 2. Sequencing analysis for genotypes of the p73 G4C14-to-A4T14 polymorphism. (A) GC/GC, (B) GC/AT and (C) AT/AT genotypes. The arrows indicate the base changes at the nucleotide position.

products are shown in Fig. 2. As shown in Fig. 1, the GC allele yielded two bands of 428 and $193 \mathrm{bp}$, the AT allele yielded two bands of 428 and $270 \mathrm{bp}$ and the GC/AT heterozygosity yielded three bands of 428, 270 and $193 \mathrm{bp}$. In order to ensure the accuracy of the genotyping data, the data was confirmed by direct sequencing analysis by Sangon Biotech Co., Ltd.) (Fig. 2). SPSS 17.0 software was used to analyze the p73 genotypes and the results are shown in Table II.

Association between p73 polymorphisms and NSCLC risk. The present study aimed to determine whether the p73 G4C14-to-A4T14 polymorphism was associated with susceptibility to NSCLC. The genotypes and allele distributions of the p73 polymorphism in patients and controls are shown in Table II; genotype frequencies in patients and controls were in accordance with the Hardy-Weinberg equilibrium. The results demonstrated a significant association between AT/AT genotype and a reduced risk of NSCLC $(\mathrm{P}=0.037 ; \mathrm{OR}=0.481 ; 95 \% \mathrm{CI}=0.240-0.965)$ compared with the $\mathrm{GC} / \mathrm{GC}$ genotype. In addition, patients carrying the AT allele (GC/AT or AT/AT) had a significantly decreased risk of NSCLC $(\mathrm{P}=0.038 ; \mathrm{OR}=0.713 ; 95 \% \mathrm{CI}=0.517-0.983)$ 
Table II. Association between p73 polymorphisms and risk of non-small cell lung cancer.

\begin{tabular}{|c|c|c|c|c|}
\hline \multirow[b]{2}{*}{ Genotype } & \multirow{2}{*}{$\frac{\text { Cases (\%) }}{n=186}$} & \multicolumn{3}{|l|}{ Controls $(\%)$} \\
\hline & & $\mathrm{n}=198$ & P-value & OR $(95 \% \mathrm{CI})$ \\
\hline $\mathrm{GC} / \mathrm{GC}$ & $108(58.1)$ & $104(52.5)$ & - & 1 \\
\hline GC/AT & $68(36.6)$ & $68(34.3)$ & 0.864 & $0.963(0.626-1.481)$ \\
\hline $\mathrm{AT} / \mathrm{AT}$ & $10(5.4)$ & $26(13.1)$ & 0.010 & $0.370(0.170-0.806)$ \\
\hline GC allele & $284(76.3)$ & $276(69.7)$ & - & 1 \\
\hline AT allele & $88(23.7)$ & $120(30.3)$ & 0.038 & $0.713(0.517-0.983)$ \\
\hline
\end{tabular}

OR and P-values were adjusted for age, gender and smoking status. OR, odds ratio; 95\% CI, 95\% confidence interval.

Table III. Association between p73 polymorphisms and clinicopathological parameters of non-small cell lung cancer.

\begin{tabular}{lrcccr}
\hline Variable & $\mathrm{n}(\%)$ & $\mathrm{GC} / \mathrm{GC}(\%)$ & $\mathrm{GC} / \mathrm{AT}(\%)$ & AT/AT (\%) & P-value \\
\hline Histological (n=174) & & & & & 0.410 \\
$\quad$ Squamous cell & $116(66.7)$ & $70(60.3)$ & $38(32.8)$ & $8(6.9)$ & \\
$\quad$ Adenocarcinoma & $58(33.3)$ & $32(55.2)$ & $24(41.4)$ & $2(3.4)$ & 0.192 \\
Tumor stage (n=186) & $150(80.6)$ & $90(60)$ & $54(36)$ & $6(4)$ & \\
$\quad$ I+II & $36(19.4)$ & $18(50)$ & $14(38.9)$ & $4(11.1)$ & 0.298 \\
III+IV & & & & & \\
Lymph node metastasis (n=186) & $48(25.8)$ & $24(50)$ & $22(45.8)$ & $2(4.2)$ & $8(5.8)$ \\
$\quad$ Yes & $138(74.2)$ & $84(60.9)$ & $46(33.3)$ & \\
No & &
\end{tabular}

compared with that of the p73 GC allele group, which indicated that the AT allele may be a protective factor against NSCLC.

Association between 73 polymorphisms and clinicopathological parameters. The association between p73 SNPs and the clinicopathological features of NSCLC is depicted in Table III. The results revealed that there was no significant association between the $\mathrm{p} 73$ genotypes and tumor histological type $(\mathrm{P}=0.410)$, tumor stage $(\mathrm{P}=0.192)$ or lymph node metastasis $(\mathrm{P}=0.298)$.

\section{Discussion}

The p73 gene is known to activate the promoters of certain genes that are responsive to $\mathrm{p} 53$, including those involved in cell cycle control, DNA repair and apoptosis; therefore, p73 attenuates cell growth through the induction of apoptosis or G1 cell cycle arrest in a manner comparable to that of the p53 gene (2). Although the accurate functional association between the risk of cancer and p73 G4C14-to-A4T14 genetic variants remains to be elucidated, it was proposed that the change of GC to AT may result in the formation of a stem-loop structure and may therefore influence p73 translation efficiency and gene expression (2).

Previous studies have provided evidence to suggest that the p73 gene has a crucial role in the pathogenesis of various types of cancer, such as lung cancer (6-9). It is therefore plausible that an association exists between the p73 G4C14-to-A4T14 polymorphism and the incidence of lung cancer. p73 is a member of the p53 family of tumor suppressor genes, which has two distinct promoters that may be expressed in different $\mathrm{N}$-terminal isoforms (20). The transactivation proficient isoform (TAp73) has pro-apoptotic functions, whereas the $\mathrm{N}$-terminally truncated isoform- $\Delta \mathrm{N}$ p73 exerts an anti-apoptotic effect. It has therefore been proposed that the altered expression ratio of $\Delta \mathrm{N}$ :TA may have a role in the pathogenesis of certain types of cancers (21-24), such as lung cancer (8). As indicated by the role of TAp73 in cancer development, it was hypothesized that individuals with the AT allele may have

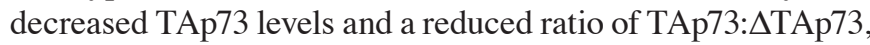
which may result in an increased susceptibility to lung cancer.

The p73 gene was suggested to be a susceptibility candidate gene for lung cancer. However, the results of previous studies regarding the association between p73 G4C14-to-A4T14 polymorphism and susceptibility to lung cancer are inconsistent $(10,15-18)$. Two previous studies observed that the AT allele was associated with a significantly increased incidence of lung cancer compared with the GC/GC allele in a Northern Chinese population (10) and a non-Hispanic Caucasian population (16). By contrast, one study reported a marked association between the AT allele and the decreased risk of developing lung cancer in a Chinese population (15), which is consistent with the results of the present study. However, a 
further two studies failed to identify any associations between the p73 G4C14-to-A4T14 polymorphism and the risk of lung cancer in Korean and Japanese populations $(17,18)$. These findings suggested that the role of the p73 G4C14-to-A4T14 polymorphism may vary between different ethnic populations.

In the present case-control study, it was investigated whether the $\mathrm{p} 73 \mathrm{G} 4 \mathrm{C} 14$-to-A4T14 polymorphism was associated with the risk of NSCLC in a Chinese population. The results revealed that the genotype distribution was markedly different between the 186 NSCLC patients and the 198 healthy controls; this therefore indicated that the p73 AT/AT genotype was associated with a decreased risk of NSCLC. In addition, no significant association was detected between p73 gene SNPs and tumor histological type, tumor stage or lymph node metastasis of NSCLC. Thus, whether the p73 gene polymorphism affected the occurrence of lung cancer requires further verification in terms of clinical development and survival.

In conclusion, the results of the present study indicated that the p73 AT allele may be a reduced genetic risk factor for the tumorigenesis and development of NSCLC in Chinese patients. Further studies are required in order to confirm the association between p73 polymorphism and the risk of lung cancer. However, if confirmed by subsequent studies, this genetic risk factor may be valuable for explaining the pathogenesis of lung cancer, which may contribute to future cancer diagnosis techniques and therapies.

\section{Acknowledgements}

The present study was supported by grants from the National Natural Science Foundation of China (no. 61171061), the Natural Science Foundation of Hunan Province of China (nos. 12JJ4082 and 14JJ2049), the Outstanding Youth Project Supported by Scientific Research Fund of Hunan Provincial Education Department (no. 14B050) and the Natural Science Foundation of Hunan University of Technology (no. 2013HZX04).

\section{References}

1. Jemal A, Bray F, Center MM, et al: Global cancer statistics. CA Cancer J Clin 61: 69-90, 2011.

2. Kaghad M, Bonnet H, Yang A, et al: Monoallelically expressed gene related to $\mathrm{p} 53$ at $1 \mathrm{p} 36$, a region frequently deleted in neuroblastoma and other human cancers. Cell 90: 809-819, 1997.

3. Bénard J, Douc-Rasy S and Ahomadegbe JC: TP53 family members and human cancers. Hum Mutat 21: 182-191, 2003.

4. Moll UM, Erster S and Zaika A: p53, p63 and p73 - solos, alliances and feuds among family members. Biochim Biophys Acta 1552: 47-59, 2001.
5. Shibukawa K, Miyokawa N, Tokusashi Y, et al: High incidence of chromosomal abnormalities at lp36 and 9p21 in early-stage central type squamous cell carcinoma and squamous dysplasia of bronchus detected by autofluorescence bronchoscopy. Oncol Rep 22: 81-87, 2009.

6. Uramoto H, Sugio K, Oyama T, et al: Expression of the p53 family in lung cancer. Anticancer Res 26: 1785-1790, 2006.

7. Liu K, Zhan M and Zheng P: Loss of p73 expression in six non-small cell lung cancer cell lines is associated with $5^{\prime} \mathrm{CpG}$ island methylation. Exp Mol Pathol 84: 59-63, 2008.

8. Lo Iacono M, Monica V, Saviozzi S, et al: p63 and p73 isoform expression in non-small cell lung cancer and corresponding morphological normal lung tissue. J Thorac Oncol 6: 473-481, 2011.

9. Nyman U, Sobczak-Pluta A, Vlachos P, et al: Full-length p73alpha represses drug-induced apoptosis in small cell lung carcinoma cells. J Biol Chem 280: 34159-34169, 2005.

10. Zhang X, Li X, Wu Z, et al: The p73 G4C14-to-A4T14 polymorphism is associated with risk of lung cancer in the Han nationality of North China. Mol Carcinog 52: 387-391, 2013.

11. Tokuchi Y, Hashimoto T, Kobayashi Y, et al: The expression of p73 is increased in lung cancer, independent of p53 gene alteration. Br J Cancer 80: 1623-1629, 1999.

12. Li Q, Athan E, S, Wei M, et al: TP73 allelic expression in human brain and allele frequencies in Alzheimer's disease. BMC Med Genet 5: 5-14, 2004.

13. Li GJ, Sturgis EM, Wang LE, et al: Association of a p73 exon 2 G4C14-to-A4T14 polymorphism with risk of squamous cell carcinoma of the head and neck. Carcinogenesis 25: 1911-1916, 2004.

14. Ryan BM, McManus R, Daly JS, et al: A common p73 polymorphism is associated with a reduced incidence of oesophageal carcinamo. Br J Cancer 85: 1499-1503, 2001.

15. Hu Z, Miao X, Ma H, et al: Dinucleotide polymorphism of p73 gene is associated with a reduced risk of lung cancer in a Chinese population. Int J Cancer 114: 455-460, 2005.

16. Li G, Wang LE, Chamberlain RM, et al: p73 G4C14-to-A4T14 polymorphism and risk of lung cancer. Cancer Res 64: 6863-6866, 2004.

17. Choi JE, Kang HG, Chae MH, et al: No association between $\mathrm{p} 73$ G4C14-to-A4T14 polymorphism and the risk of lung cancer in a Korean population. Biochem Genet 44: 533-540, 2006.

18. Hiraki A, Matsuo K, Hamajima N, et al: Different risk relations with smoking for non-small cell lung cancer: Comparison of TP53 and TP73 genotypes. Asian Pac J Cancer Prev 4: 107-112, 2003.

19. Lee KE, Hong YS, Kim BG, et al: p73 G4C14 to A4T14 polymorphism is associated with colorectal cancer risk and survival. World J Gastroenterol 16: 4448-4454, 2010.

20. Melino G, De Laurenzi V and Vousden KH: p73: Friend or foe in tumorigenesis. Nat Rev Cancer 2: 605-615, 2002.

21. Grob TJ, Novak U, Maisse C, et al: Human delta Np73 regulates a dominant negative feedback loop for TAp73 and p53. Cell Death Differ 8: 1213-1223, 2001.

22. Ishimoto O, Kawahara C, Enjo K, et al: Possible oncogenic potential of DeltaNp73: A newly identified isoform of human p73. Cancer Res 62: 636-641, 2002.

23. Tomasini R, Tsuchihara K, Wilhelm M, et al: TAp73 knockout shows genomic instability with infertility and tumor suppressor functions. Genes Dev 22: 2677-2691, 2008.

24. Concin N, Becker K, Slade N, et al: Transdominant DeltaTAp73 isoforms are frequently up-regulated in ovarian cancer. Evidence for their roles as epigenetic p53 inhibits in vivo. Cancer Res 64: 2449-2460, 2004. 